\title{
REFRESMENT Pharmacological management of antipsychotic-induced akathisia: an update and treatment algorithm
}

\author{
Ketan Dipak Jethwa
}

\author{
Ketan Dipak Jethwa is currently \\ a CT1 in medicine at Nottingham \\ University Hospitals NHS Trust. He \\ was previously Academic Clinical \\ Fellow in General Psychiatry \\ at the University of Warwick. \\ Correspondence Dr K.D. \\ Jethwa, Nottingham University \\ Hospitals NHS Trust, Nottingham \\ City Hospital, Hucknall, Nottingham \\ NG5 1PB, UK. Email: ketan.jethwa@ \\ nuh.nhs.uk
}

\begin{abstract}
SUMMARY
Akathisia is a common and distressing side-effect of antipsychotic medication. The treatment of this condition is difficult and options are limited. This review of the literature was performed to develop an evidence-based algorithm for the management of antipsychotic-induced akathisia. There is limited high-level evidence to support the use of anticholinergics. Centrally acting $\beta$-adrenergic antagonists are potential treatments, although their use is limited by troublesome side-effects. Long-acting benzodiazepines and mirtazapine are potential well-tolerated treatments for the acute management of akathisia.

\section{DECLARATION OF INTEREST}

None
\end{abstract}

Akathisia is a common movement disorder characterised by psychomotor agitation that includes a sense of inner restlessness and nonpurposive motor activity. These subjective and objective components of the syndrome are commonly assessed using the Barnes Akathisia Rating Scale (also known as the Barnes Akathisia Scale; Barnes 1989). Akathisia is frequently associated with the use of antipsychotics (neuroleptics), but it can also complicate the use of selective serotonin reuptake inhibitors (SSRIs). Other causes of akathisia include anxiety disorders, drug withdrawal or discontinuation states, early serotonin syndrome, restless legs syndrome, irondeficiency anaemia and endocrinopathies. It is therefore important to find the underlying cause by identifying drug precipitants and associated psychopathology and excluding general medical causes.

The pathophysiology of akathisia is not fully understood, but a combination of hypodopaminergic and hyperserotonergic neurotransmission may be implicated (Poyurovsky 2001). This hypothesis is supported by the emergence of akathisia following the initiation of antipsychotics or SSRIs, but also from the observation that dopamine agonists can alleviate psychomotor agitation associated with restless legs syndrome (Garcia-Borreguero 2011).

Akathisia can have a significant negative impact on quality of life and is associated with increased suicidality (Seemüller 2012). Treatment of this distressing condition is difficult and treatment options are limited. I therefore carried out a review of the literature to devise an evidence-based treatment algorithm.

\section{Treatment strategies}

The treatment of akathisia may be divided into two components:

- change in antipsychotic medication

- addition of another therapeutic agent.

Pragmatic changes to the antipsychotic regimen include:

- reducing the dose (or withdrawal) of the antipsychotic

- switching to a low-potency first-generation antipsychotic or a second-generation antipsychotic with a low potential for causing akathisia.

In practice, use of this strategy is limited by the potential for deterioration in mental state. In addition, analysis of the Clinical Antipsychotic Trials of Intervention Effectiveness (CATIE) data found similar rates of akathisia among patients prescribed first-and second-generation antipsychotics (Lieberman 2005; Miller 2008). Therefore, the identification of safe and effective medications to manage akathisia is imperative.

\section{Literature search}

A search of the Cochrane Library using the search term 'akathisia' found three Cochrane reviews of centrally acting $\beta$-adrenergic antagonists (betablockers), anticholinergics and benzodiazepines. I then performed a MEDLINE search (MeSH terms: akathisia, treatment; limits: English language, randomised controlled trial, human) to identify additional double-blind randomised placebo- 
controlled trials using these three agents. The results of this literature search are summarised in the following sections.

\section{Centrally acting $\beta$-adrenergic antagonists}

A Cochrane review in 2004 identified three randomised placebo-controlled trials $(n=51)$ of centrally acting beta-blockers (Lima 2004a). The authors stated that there were insufficient data to recommend their use for the management of akathisia. In two $48 \mathrm{~h}$ studies using propranolol (60-80 mg), none of the 31 participants experienced full remission of akathisia (Irwin 1988; Kramer 1988), but the drug was well tolerated and no one discontinued treatment. In a $72 \mathrm{~h}$ trial $(n=20)$ no statistically significant difference was found between the novel centrally acting beta-blocker ICI-118,551 and placebo for the outcome 'no change/worse' (Adler 1989). No adverse events were reported. The findings of Lima et al's review and meta-analysis (Lima 2004a) are limited by the small data-set and short trial duration. There were also important methodological limitations: not all of the trials were explicit about the process of randomisation or the maintenance of doubleblind conditions.

My MEDLINE search found one additional randomised placebo-controlled trial $(n=90)$ (Poyurovsky 2006) since the publication of the 2004 Cochrane review. This involved a 7-day double-blind trial of propranolol $(80 \mathrm{mg})$ and used the Barnes Akathisia Scale (BAS) to assess treatment response. This trial had two treatment arms (propranolol and mirtazapine) compared with placebo. The results of the mirtazapine arm are discussed below. Propranolol resulted in a significant reduction in akathisia symptoms (BAS score improved by $29 \%$ over the trial period). However, $27 \%$ of the participants discontinued propranolol because of non-response and 17\% withdrew because of hypotension and bradycardia.

\section{Anticholinergics}

A Cochrane review (Lima 2004b) systematically searched the literature to identify randomised placebo-controlled trials investigating the role of anticholinergics in antipsychotic-induced akathisia. The authors found no relevant trials and concluded that there was no reliable evidence to support or refute the use of anticholinergic agents.

My MEDLINE search yielded one shortterm placebo-controlled trial (Baskak 2007): this reported no difference in outcome between intramuscular biperiden and placebo.

The use of anticholinergics is also limited by an increased risk of related side-effects.

\section{Benzodiazepines}

A Cochrane review (Lima 2002) identified two randomised placebo-controlled trials $(n=27)$ using clonazepam (0.5-2.5 mg daily), a longacting benzodiazepine (Kutcher 1989; Pujalte 1994). Overall, clonazepam was more effective in achieving 'partial remission' by day 7, with a number needed to treat (NNT) of 1.2 (Lima 2002). 'Partial remission' was defined as an improvement in BAS or Extrapyramidal Symptom Rating Scale (ESRS) scores by at least 50\%. No adverse events or withdrawals were reported in both trials. Again, the findings of Lima et al's review were limited by the small data-set and not all of the trials being explicit about the randomisation process or the maintenance of double-blind conditions. Tolerance and dependence are also considerations, especially when thinking about long-term treatment.

My MEDLINE search found no relevant randomised placebo-controlled trials since the Cochrane review.

\section{Mirtazapine: a novel therapeutic option}

The MEDLINE search found a number of small randomised controlled trials investigating other akathisia treatments, including trazodone (Stryjer 2010), vitamin $B_{6}$ (Lerner 2004; Miodownik 2006) and mianserin (Poyurovsky 1999), each of which demonstrated therapeutic benefit. However, there appears to be a growing body of data suggesting that mirtazapine, a presynaptic $\alpha_{2}$ receptor and specific serotonin antagonist, may have a greater role to play in the management of akathisia. The search also identified two randomised placebocontrolled trials using mirtazapine (total sample: 116), and these are discussed below. It is important to note that mianserin and mirtazapine have similar pharmacological actions.

A double-blind randomised placebo-controlled trial of mirtazapine $(n=26)$ of 5 days' duration was performed in 2003 (Poyurovsky 2003). Symptom severity was assessed using the BAS. The randomisation sequence was stated: 13 patients received the active drug (mirtazapine $15 \mathrm{mg}$ daily); $54 \%$ in the treatment group had a reduction in BAS scores of $\geq 2$. This improvement in symptoms was statistically significant $(P=0.004)$. Complete remission of symptoms occurred in $31 \%$ of the mirtazapine group; $23 \%$ of the group withdrew from the study before the day 3 assessment because of non-response or sedation.

A second randomised placebo-controlled trial $(n=90)$ of 7 days' duration was performed in 2006 (Poyurovsky 2006). The trial had two active groups (mirtazapine $15 \mathrm{mg}$ daily and propranolol $40 \mathrm{mg}$ daily) and a placebo group. The randomisation 


\begin{tabular}{|l|l|}
\hline First-line management & Mirtazapine $15 \mathrm{mg}$ daily \\
\hline Second-line management & Clonazepam $1 \mathrm{mg}$ daily \\
\hline Third-line management & $\begin{array}{l}\text { Propranolol } 80 \mathrm{mg} \text { daily, } \\
\text { procyclidine } 5 \mathrm{mg} \text { daily (if Parkinsonian symptoms present) }\end{array}$ \\
\hline
\end{tabular}

- Treatment should be limited to 4 weeks for each agent before switching (if symptoms persist) or discontinuation (following symptomatic resolution)

- In addition to pharmacotherapy, patients should have regular risk assessments and structured psychosocial support and psychoeducation to help them manage their symptoms and aid medication adherence

Evidence-based treatment algorithm for the management of antipsychotic-induced akathisia.

sequence was stated. Symptom severity was again assessed using the BAS. I discussed the propranolol results above. In the mirtazapine group $(n=30)$, the drug resulted in a statistically significant reduction in akathisia symptoms (BAS score improved by $34 \%$ over the trial period; $P=0.012$ ). Ten patients in the mirtazapine group (33\%) experienced a complete remission in symptoms; $36.7 \%$ experienced drowsiness, but this did not precipitate study withdrawal; $23 \%$ discontinued treatment early because of non-efficacy.

\section{An evidence-based treatment algorithm}

As this literature search shows, the evidence at present is limited and there is need for further evaluative studies. In the meantime, I propose the treatment algorithm shown in Fig. 1, which is based on the available evidence, to maximise therapeutic effect and reduce adverse effects. Note that, in keeping with best practice, this includes psychosocial support and psychoeducation to help patients manage their symptoms and adhere to their medication.

\section{Acknowledgements}

I would like to thank Professor Peter Liddle (Professor of Psychiatry, University of Nottingham) and Dr Verghese Joseph (Stamford Resource Centre) for reviewing the first draft of this article.

\section{References}

Adler L, Duncan E, Angrist B, et al (1989) Effects of a specific beta 2-receptor blocker in neuroleptic-induced akathisia. Psychiatry Research, 27: 1-4.

Barnes TR (1989) A rating scale for drug-induced akathisia. British Journal of Psychiatry, 154: 672-6.

Baskak B, Atbasoglu EC, Ozguven HD, et al (2007) The effectiveness of intramuscular biperiden in acute akathisia: a double-blind, randomized, placebo-controlled study. Journal of Clinical Psychopharmacology, 27: 289-94.

Garcia-Borreguero D, Stillman P, Benes H, et al (2011) Algorithms for the diagnosis and treatment of restless legs syndrome in primary care. BMC Neurology, 11: 28

Irwin M, Sullivan G, Van Putten T (1988) Propranolol as a primary treatment of neuroleptic-induced akathisia. Hillside Journal of Clinical Psychiatry, 10: 244-50.

Kramer MS, Gorkin RA, DiJohnson C, et al (1988) Propranolol in the treatment of neuroleptic-induced akathisia (NIA) in schizophrenics: a double-blind, placebo-controlled study. Biological Psychiatry, 24: 823-7.

Kutcher S, Williamson P, MacKenzie S, et al (1989) Successful clonazepam treatment of neuroleptic-induced akathisia in older adolescents and young adults: a double-blind, placebo-controlled study. Journal of Clinical Psychopharmacology, 9: 403-6.

Lerner V, Bergman J, Statsenko N, et al (2004) Vitamin B6 treatment in acute neuroleptic-induced akathisia: a randomized, double-blind, placebo-controlled study. Journal of Clinical Psychiatry, 65: 1550-4.

Lieberman JA, Stroup TS, McEvoy JP, et al (2005) Effectiveness of antipsychotic drugs in patients with chronic schizophrenia. New England Journal of Medicine, 353: 1209-23.

Lima AR, Soares-Weiser K, Bacaltchuk J, et al (2002) Benzodiazepines for neuroleptic-induced acute akathisia. Cochrane Database of Systematic Reviews, 1: CD001950

Lima AR, Bacaltchuk J, Barnes TR, et al (2004a) Central action betablockers versus placebo for neuroleptic-induced acute akathisia. Cochrane Database of Systematic Reviews, 4: CD001946.

Lima AR, Weiser KV, Bacaltchuk J, et al (2004b) Anticholinergics for neuroleptic-induced acute akathisia. Cochrane Database of Systematic Reviews, 1: CD003727

Miller DD, Caroff SN, Davis SM, et al (2008) Extrapyramidal side-effects of antipsychotics in a randomised trial. British Journal of Psychiatry, 193 : 279-88.

Miodownik C, Lerner V, Statsenko N, et al (2006) Vitamin B6 versus mianserin and placebo in acute neuroleptic-induced akathisia: a randomized, double-blind, controlled study. Clinical Neuropharmacology, 29: $68-72$

Poyurovsky M, Shardorodsky M, Fuchs C, et al (1999) Treatment of neuroleptic-induced akathisia with the 5-HT2 antagonist mianserin: double-blind, placebo-controlled study. British Journal of Psychiatry, 174: 238-42.

Poyurovsky M, Weizman A (2001) Serotonin-based pharmacotherapy for acute neuroleptic-induced akathisia: a new approach to an old problem. British Journal of Psychiatry, 179: 4-8.

Poyurovsky M, Epshtein S, Fuchs C, et al (2003) Efficacy of low-dose mirtazapine in neuroleptic-induced akathisia: a double-blind randomized placebo-controlled pilot study. Journal of Clinical Psychopharmacology, 23: $305-8$.

Poyurovsky M, Pashinian A, Weizman R, et al (2006) Low-dose mirtazapine: a new option in the treatment of antipsychotic-induced akathisia. A randomized, double-blind, placebo- and propranololcontrolled trial. Biological Psychiatry, 59: 1071-7.

Pujalte D, Bottaï T, Huë B, et al (1994) A double-blind comparison of clonazepam and placebo in the treatment of neuroleptic-induced akathisia. Clinical Neuropharmacology, 17: 236-42.

Seemüller F, Schennach R, Mayr A, et al (2012) Akathisia and suicidal ideation in first-episode schizophrenia. Journal of Clinical Psychopharmacology, 32: 694-8.

Stryjer R, Rosenzcwaig S, Bar F, et al (2010) Trazodone for the treatment of neuroleptic-induced acute akathisia: a placebo-controlled, doubleblind, crossover study. Clinical Neuropharmacology, 33: 219-22. 